\title{
Dispatch
}

\section{Is it Resolved? One Story of Academic Contrapower Harassment and Cyberbullying}

\author{
ANONYMOUS $^{1}$
}

I am a professor at a Canadian university who recently experienced more than a year of harassment by an undergraduate student in an upper year class. During this period, I tried to connect with professors who had similar experiences, but I could not find anyone. Nor could I find more than a handful of detailed online personal accounts or academic articles relating to student-on-faculty harassment, despite clear evidence that it is not uncommon. In a study of 524 professors across 100 American colleges and universities, Lampman (2012, p. 184) found that "91\% reported at least one act of student incivility/bullying, 25\% experienced at least one sexual behavior from a student, and one to two percent said a student had used or threatened them with violence in the past year." Although Lampman's study, like several others, incorporates quotations from faculty interviews about their experiences, longer and more detailed experiential accounts are rare. Yet, learning how other faculty members experience and deal with student harassment might have helped me navigate my situation. In this dispatch I offer the sort of narrative I was searching for during and after this difficult period of my life, in hopes that it will be useful to others in similar or analogous circumstances. Beyond a simple recounting of the details of my experience, I aim to provide insight into what others in comparable circumstances might feel, to identify some challenges that may be avoided, and to add texture to understandings of student-on-faculty harassment.

Student-on-faculty harassment is a form "academic contrapower harassment" (ACPH), which "occurs when someone with seemingly less power in an educational setting [e.g., a student] harasses someone more powerful [e.g., a professor]" (Lampman, Crew, Lowery, \& Tompkins, 2016,

\footnotetext{
${ }^{1}$ Editor's note: In this case, Studies in Social Justice respects the author's preference to remain anonymous. Any correspondence may be sent to the address below, from where it will be redirected to the author.
} 
p. 169). Studies demonstrate that faculty members across genders are harassed by students, through a variety of means, and for a variety of reasons (Blizard, 2016; Lampman, 2012; Lampman et al., 2016; MacLennan, 2002). For example, Blizard's (2016) study of one Canadian university found that student-on-faculty cyberbullying mainly, but not exclusively, stems from students' dissatisfaction with their grades. Although some research explores contrapower harassment and bullying, including its negative impacts on faculty members, public attention and university resources largely address noncontrapower forms of harassment, such as faculty-on-student harassment and faculty-on faculty harassment. This focus overlooks how feminized, racialized, young and less experienced and credentialed faculty are especially vulnerable to harassment by students (Lampman, 2012), thereby neglecting the multiple ways that power operates within the university. Claudia Lampman, ACPH expert and psychology professor, commented in a recent interview that women and minority professors are "more likely to be threatened and challenged by students - usually white males" (Lampman, quoted in Flaherty, 2018). Failure to confront such forms of harassment reproduces a dualistic and unnuanced understanding of power, in which the categories of "students" and "professors" are homogenized, and power is thought to operate only in traditionally imagined, top-down ways within the institutional hierarchy. As MacLennan (2002, p. 10) argues,

As professionals, of institutional cultures, we must recognize the complex nature of human relations in the workplace and establish more humane approaches to resolving the very real problem of harassment; to do this, we must recognize that harassment is not a simple, one-way behavior perpetrated only by those who are recognized to hold institutional power.

Many Canadian universities have begun to reflect on and refashion their formal harassment policies and support services, especially in the era of "Me Too." Survivors are publicly telling their stories of harassment on campus, which affects universities' reputations. In the same moment, we have also witnessed institutional attempts to deflect accusations of harassment and silence students who disclose their experiences. Within this political farrago, harassment is usually imagined to only involve particular university members; we hear little about students' harassment of professors (Flaherty, 2018). Only a few first-hand published accounts exist, and even fewer from Canadian professors (e.g., Anonymous, 2017; MacLennan, 2000). I would argue that ignorance of any form of harassment is antithetical to the values of critical thinking and social justice, values that are aspirational to many within the university. From my vantage, efforts to tackle student-on-faculty harassment are therefore extensions, rather than diminishments, of social justice. Here I add to the growing conversation about university-based harassment by demonstrating some of the complexity of ACPH from an insider's perspective. I also try to disrupt some conventional assumptions about how power can operate within student-professor relations, especially in 
the age of social media. Finally, I offer some broad stroke suggestions about moving forward.

In the account that follows, I have excluded numerous identifying details, weighing their importance against the need to protect myself from further provocation by the student. I am a tenured faculty member and a woman. As research demonstrates, gender, race, ethnicity, age, and university rank inform student-on-faculty harassment (see Lampman et al., 2016). To maintain my anonymity, I do not comment at length on how these aspects of my social positionality shaped my experience, and I have kept certain details intentionally vague.

While my social location influenced my experience of the harassment, the student's social location also affected my response to their behaviour. They struggle with mental health issues and are socially marginalized in multiple ways. I wanted to be cognizant of these dynamics when handling the situation. As such, I was hesitant to approach the security office, and I was reluctant to involve the police. Early in the harassment I turned down one administrator's offer to have a security officer monitor the classroom. I felt that pursuing the more formal and institutionalized routes for harassment management, such as security offices and police, can gloss over complex issues of power, including the marginalization of those involved. While marginalization does not excuse inappropriate behaviour, it figures into how I experienced and handled the unwanted interactions.

Although the effects of the harassment on me were manifold, they can be distilled into two main concerns: my physical safety and my career. Like most academics, I devoted many years to research specialization with the hope of becoming a professor. I feared that my time and labour commitment, and my professional reputation, would be jeopardized or, at the very least, damaged by the student's libelous and delusional online messages. I worried that these messages would eventually metastasize into an all-out smear campaign. Undoubtedly, the harassment was frightening, but my concern that it could turn into something far worse - a physical assault or public slander exacerbated my fear.

In the first period of the harassment, about midway through the term, when the student began following me on social media, then sporadically unfollowed and refollowed me, I convinced myself that their behaviour was inconsequential. But some time later, I noticed the student had searched through years of old social media photos, and "liked" those that included me. I showed the Department Chair the string of "likes" and asked, "This is creepy, right?" They agreed, so we made an appointment with the Dean to formally document the behaviour and discuss my options should the situation get worse.

Unfortunately, as the weeks progressed, the student began messaging me over social media, sending progressively more angry and distressing tirades while simultaneously disclosing romantic feelings for me. They expressed indignation that I refused to engage with them beyond institutional email, and 
abnegation that they regarded as a personal affront, stifling the kinds of things we might discuss. I blocked their accounts, only for them to create "decoy" ones from which to send more agitated and acidic messages. They wrote about how hurt they were, how awful I was for ignoring them, and how shocked they were at my ostensible disregard for their feelings. The student interpreted my continued lack of response, which was recommended by my Chair, as action through non-action; they read it as hostility.

By this time, the term was not yet complete, and final exams were imminent. Although my Chair cancelled my last lecture, navigating the exam spurred fresh anxieties as I struggled with what to tell my teaching assistants and how to manage my fear of further contact with the student. It seemed futile to press charges or even go to the police when the messages did not contain direct threats and framed the student as a victim of unrequited attention and affection. Social media messages would arrive and then be erased; some platforms allow users to delete messages if the recipient has not clicked "accept" on a message from an unknown user. However, given that the recipient can read the message without "accepting" it, I saw these notes pop up and then vanish either immediately or within a few hours. Fortunately, I was able to take screenshots (i.e., photos of the screen content) before the student had a chance to delete their messages, which allowed me to compile a comprehensive dossier that I would later submit to my Chair, Dean, faculty association, and human rights and security offices.

The student's social media messages were coupled with increasing institutional contact, in which they frequently requested meetings to review assignments and course material, with numerous additional reminders of these upcoming "by appointment" meetings and follow up emails after we met. Additionally, the student attended all my office hours. Indeed, the student sought out any available opportunity to spend time with me: from cornering me after lecture to talk, to loitering near my office, to unexpectedly popping by for impromptu meetings when my door was open. While these behaviours do not constitute harassment, when paired with their ongoing vitriolic social media messages, these other engagements took on a decidedly more sinister tone.

My first approach was to try to bypass all unnecessary contact, keeping my door shut and staying off campus as much as possible. In the case of lectures, I ensured a friend would be waiting for me after class. A university administrator noted that the student was "holding me hostage" as I contorted myself to avoid the student. The Chair, Dean, and I hoped the student would stop contacting me once the class finished. As the student's online messages grew more caustic and accusatory, and continued past the end of the term, my Chair and I met with the security office. Prior to meeting, we were unclear what help the office could offer, especially as the student was no longer registered with the university.

To our surprise, my Chair and I learned that security personnel regularly deal with cases of contrapower harassment, which, although disheartening, 
left me feeling relieved not to be alone. The office presented us with a set of protocols they typically offer to harassed professors, such as a panic button for my office and a direct emergency line.

Alongside these resources that ensured my physical safety on campus, security officers suggested I ask colleagues near my office to alert me if they spotted the student in the department. Perhaps most importantly, they urged me to send the student a "no contact" letter through their office, which they maintained would close the case. Although I feared the letter might actually worsen the harassment, I took their advice.

Breaking the no contact request, the student sent the security office and me a furious response. They only vaguely acknowledged that they had sent inappropriate messages through official means, which represented a small portion of the harassment.

Security officers responded to the no contact violation, but, contrary to my wishes, they neglected to indicate they had screenshots of the social media messages. They argued that mentioning these images would invite the student to engage further, by giving them more information to challenge or deny. Yet, by not explicitly mentioning the screenshots, which captured the bulk of alarming messages, the security office left the student's specific transgressions open to interpretation. This omission allowed the student to believe that the no contact request was simply a result of their institutional emails. They disregarded what I felt was necessary to increase my sense of safety, with only a cursory rationale. I wanted the student to know that their communications were fully documented and submitted to university authorities, with the hope that this knowledge would deter them from sending further messages. Unequivocally, social media provided an essential part of the student's access to me, which profoundly shaped how the harassment progressed; yet, the security office faltered, as these sorts of online engagements fell outside their knowledge. I did not anticipate the struggle I would have to explain how social media platforms function to the people tasked with addressing harassment at the university. Despite their unfamiliarity with online harassment, once I handed over my file every action was vetted through the security office's formal processes and human resources, and my requests were largely discounted. After we sent the no contact letter, security told me to "let it go" and stop worrying. I was told they would mediate the conflict. A process that was meant to protect me, and had initially provided some assurance, now added to my unease.

In what turned out to be the end of the harassment, security called me to communicate their increased awareness regarding the malleable dimensions of cyber-harassment. They finally understood that the student could continue to create decoy social media accounts to maintain contact with me. Flabbergasted by the plasticity of social media to serve nefarious ends, the head staff member exclaimed, "What do you think will happen?" Such lack of familiarity with online harassment and social media activity illuminated the need for my institution to develop policies and procedures for regulating, 
investigating, and managing student-on-faculty cyber-bullying. In my experience, the university's paucity of up-to-date information about how online modalities can shape students' malevolent access to each other and to professors made an already onerous situation even more challenging (see Blizard, 2016). Without such information, I was mostly left to address the social media dynamics on my own.

In addition to blocking the student over social media, I also deployed a series of other tactics to diffuse and deescalate the situation. After each of these efforts, friends and colleagues would ask, "Do you think it is resolved?" When checking in about how I was doing, people asked, "Is the case resolved?" The issue of resolution was raised so frequently that I learned to brace for it. While such questions are undoubtedly rooted in concern and a wish for the harassment to end, answering them proved difficult because of the ongoing nature of the harassment. As I managed my own anxieties, I also felt obligated to reassure others that I was optimistic that the harassment was over, even when I knew resolution was remarkably uncertain.

Each time I thought the harassment had finished, I was surprised by a new message, email, or maneuver used by the student to keep in contact. When I felt renewed optimism that the harassment had stopped, I was startled out of a relative calm. I learned to be vigilant and prepare for more, as allowing myself to relax intensified the extreme emotional juxtaposition. That is, I felt mentally and emotionally jolted - caught unawares - if my guard was down. The stress gnawed at me.

According to Lampman et al. (2016, p. 4), professors who experience contrapower harassment linked the harassment to significant tolls on their work and well-being:

Lampman and colleagues (2009) found that faculty experiencing ACPH reported anxiety, difficulty sleeping and concentrating, depression, fear of the student perpetrator, and stress-related illness; they also found that women faculty reported significantly more negative consequences than men faculty. DeSouza (2011) also found that faculty who had experienced ACPH reported worse job-related outcomes (including job stress, satisfaction, and turnover intent) than nonharassed faculty, although he did not find gender to be a predictor of such negative outcomes.

Blizard (2016) speculates that, given its documented long-term negative effects, student-on-faculty cyberbullying can be harmful enough to precipitate post-traumatic stress disorder and acute stress disorder. While I hope professors' mental health is a sufficient reason to put ACPH on any university's or college's radar, another impact of such behaviour is the compromised functioning of the institution itself. Given documented stress arising from ACPH, including a reported desire to leave their jobs (Lampman, Phelps, Bancroft, \& Beneke, 2009; Lampman et al., 2016), faculty's job-related outcomes are negatively affected (DeSouza, 2011). This was certainly true in my case. 
As I worked to handle the harassment, I commented to friends that I had launched an ambitious new research program called, "My Harassment Project." Exasperated and scared, I remarked to another friend that I simply did not know how to be harassed (of course, nobody does). I understood harassment primarily within the framework of students harming other students, professors harming students, and faculty harming each other. I was conceptually and analytically upended by this unexpected reversal of power. I felt entangled in a briar patch of fear and trauma, which encumbered my ability to clearly judge the best ways forward. Well meaning administrators, staff, colleagues, and friends, looked to me for guidance about how I wanted to proceed, and left me to evaluate myriad options, all in an effort (I would imagine) to be as "victim" or "survivor-centred" as possible. Without experience of such cases and the ability to parse my emotional reactions from the practical matters of strategy (what is the best next step?), I felt ill equipped to assess the multitude of choices before me. From the beginning, I did not know what resources to access, with whom to speak, or the order in which to speak to them (the faculty association first? or the Chair? the campus security office or the Dean?). I did not know what strategies to employ in addressing the problem, or how to deescalate the harassing behaviour, let alone which actions might make it worse. For example, every time I asserted a boundary, the harassment would intensify, as the student would inevitably retaliate against my attempts at self-protection.

Both administrators' and the faculty association's responses to the "unwanted contact" demonstrated that no one could identify an appropriate course of action. For example, when I approached the faculty association early in the harassment, they kindly offered sympathy, but acknowledged there were no precedents on file beyond a couple of professors who had been bullied by an entire class. While I was fortunate that my Chair and Dean took my experience seriously, even when things just felt "off," the more distinctly troubling events did not initiate a set of university protocols for handling student-on-faculty harassment.

Granted, I realize the particularity of each situation will inevitably shape the appropriate path to resolution. No single protocol can address all the specificities of each circumstance; the fluid nature of ongoing harassment requires flexible responses that are tailored to the idiosyncrasies of the circumstances. Still, there was also the unavoidable strangeness of the imbroglio that left many of my university contacts at a loss; they supported me in principle, but how that support might translate into action was sometimes nebulous, and in certain instances it became apparent that the support was related to optics and the protection of the university rather than to myself. As a result, I often felt isolated in the free fall of the harassment as my anxiety mounted.

The experience was exhausting. It set back my research agenda for months, and consumed valuable time as I sought counseling and attempted to regain my intellectual focus. Recovery is not immediate. Echoing the advice of 
Anonymous (2017), I encourage faculty who have struggled with ACPH to seek mental health supports. I also encourage academic institutions to develop widely communicated protocols that specifically name faculty as possible victims of harassment. Lampman et al. $(2016$, p. 12) provide further recommendations:

Given that women faculty in this study reported significantly more challenging and disrespectful behaviours from their students, more complex experiences of $\mathrm{ACPH}$, and more associated negative consequences than men faculty, it seems important for new faculty members - especially young women - to be instructed about how common ACPH is, what to expect, how to respond, and how to report it.

I strongly believe that faculty across seniority could greatly benefit from education concerning ACPH resources and paths to resolution.

My experience left me feeling frustrated as I navigated both my internal processing and the ecosystem of university administrators, the human rights office, the security office, human resources, the president's office, and the faculty association. I worked to organize an ongoing dialogue and agenda for action among these actors, which was emotionally draining and time consuming. The process involved creating detailed documentation, writing out the minutiae of the student's actions (i.e., time, place, type of communication), cataloguing emails/screenshots of messages, retelling the story (to keep everyone abreast of new developments, as well as others' efforts to mitigate the situation), and coordinating about a dozen or more individuals who were eventually involved. Considered together, informing and coordinating the relevant university bodies significantly compounded the stress of the harassment itself. If I had not directly experienced it, I would not have imagined the latticework of relationships and network of bureaucratic bodies I would need to mobilize to address the situation, nor the labour of synthesizing them into a coherent effort. By the harassment's denouement, I was emotionally frayed, and had lost faith in my institution's ability to adequately respond. As such, I hope to see my institution create policies and protocols that directly address student-on-faculty harassment (including cyberbulling).

As I was completing this article, colleagues suggested that I provide a checklist of steps to take if readers find themselves in similar situations. I am hesitant to offer prescriptive advice about how to adeptly handle similar ACPH situations, because each case is particular, and Canadian universities develop policies independently. Regardless of circumstance, though, I think it is paramount to keep a detailed record of the harassment, including timelines of incidents, descriptions of encounters, email trails, and screenshots. As I have noted, I was continually grateful for my detailed file, especially as each relevant university office was mobilized in the case and required such information. Fastidious documentation also made it easier to prove the harassment, and to demonstrate its severity and persistence. I am reluctant to 
say I should have gone to the security office sooner, as the efforts by the Dean and Chair likely mediated conflict with the student, and offered temporary buffers against some of the potential interactions between us. Further, as discussed, the security office lacked understanding of social media. Another complicating factor was that the harassment continued after the student was no longer registered at the university. Thus, the security office lacked jurisdiction, which limited their ability to intervene, beyond sending and then re-sending the no contact letter. As mentioned, once the student breached the terms of the letter, which clearly stated they were not to contact me through any means, the security office and then the human rights office reissued the no-contact missive. Although I did not know it at the time, these interventions would end the harassment. If they had not, I would have gone to the police to open a file and possibly apply for a peace bond.

Within Canada, a peace bond is "...used where an individual (the defendant) appears likely to commit a criminal offence, but there are no reasonable grounds to believe that an offence has actually been committed" (Government of Canada, Department of Justice, 2015). A recent discussion with a friend informed me about such a possibility. Had I known earlier, I would have considered the option. Despite the protections such bonds can afford, they may take several weeks or months to obtain, which reduces their usefulness in cases like mine. Undoubtedly, dealing with the police would have been stressful and increased my concern about escalation, but that intervention might have provided me a greater sense of safety and agency. Instead, the heightened vulnerability wore on me.

In the final month of the harassment, I sat on a friend's couch with my head in my hands, feeling wrung out as I recounted the student's latest actions. I asked for reassurance that it would be okay, that my career would not be ruined, and that this situation would eventually register as a bad but not defining experience in my life. She told me that the crisis was temporary. It was not my fault that the student was harassing me. I recalled her words as the student's behaviour persisted, and I searched for ways to resist and reclaim the experience. I made a commitment to write about it, and add further depth to public accounts of student-on-faculty harassment. I wanted to offer something valuable to professors dealing with ACPH and to encourage administrators to develop policies that explicitly address it, particularly attending to how social media broadens students' access to professors. Fortunately, I have not heard from the student in some time. It has only been within the last couple of months that I have felt some calm return to my daily life. I sincerely hope the potential isolation and stigmatization of $\mathrm{ACPH}$ are lessened for others through the recollection of experiences like mine. 


\section{References}

Anonymous. (2017, May 5). When a professor is sexually harassed by a student. Inside Higher $E d$. Retrieved from www.insidehighered.com/advice/2017/05/05/advice-faculty-memberswho-are-sexually-harassed-their-students-essay

Blizard, L. M. (2016). Faculty members' experiences of cyberbullying by students at one Canadian university: Impact and recommendations. International Research in Higher Education. 1(1), 107-124.

DeSouza, E. (2011). Frequency rates and correlates of contrapower harassment in higher education. Journal of Interpersonal Violence, 26(1), 158-188.

Flaherty, C. (2018, March 15). When students harass professors. Inside Higher Ed. Retrieved from www.insidehighered.com/news/2018/03/05/contra-power-harassment-professorsstudents-isnt-common-its-real

Government of Canada, Department of Justice. (2015). Victims' rights in Canada: Peace bonds fact sheet. Retrieved from www.justice.gc.ca/eng/cj-jp/victims-victimes/factsheetsfiches/peace-paix.html

Lampman, C. (2012). Women faculty at risk: U.S. professors report on their experiences with student incivility, bullying, aggression, and sexual attention. NASPA Journal about Women in Higher Education, 5(2), 184-208.

Lampman, C., Crew, E., Lowery, S., \& Tompkins, K. (2016). Women faculty distressed: Descriptions and consequences of academic contrapower harassment. NASPA Journal About Women in Higher Education, 9(2), 169-189.

Lampman, C., Phelps, A., Bancroft, S., \& Beneke, M. (2009). Contrapower harassment in academia: A survey of faculty experience with student incivility, bullying, and sexual attention. Sex Roles, 60, 331-346.

MacLennan, J. (2002). "Contra-power" harassment in the blackboard jungle. Sexuality \& Culture. 4(1), 75-84. 